EPJ Web of Conferences 19, 01002 (2012)

DOI: $10.1051 /$ epjconf/20121901002

(C) Owned by the authors, published by EDP Sciences, 2012

\title{
Formation of Milky Way-type stellar haloes in a $\Lambda$-CDM cosmology
}

\author{
A.S. Font ${ }^{\mathrm{a}, \mathrm{b}}$ \\ Institute of Astronomy, University of Cambridge, Madingley Road, Cambridge CB3 OHA, UK
}

\begin{abstract}
Recent observations suggest that the Milky Way stellar halo has a 'dual nature', meaning that both dissipational and dissipationless processes play a role in its build-up. The GIMIC suite of cosmological hydro-dynamical simulations show that, for Milky Way-mass haloes, in situ star formation is the dominant factor in the inner $<20-30 \mathrm{kpc}$, while tidal disruption of satellite galaxies contributes primarily to the outer regions. The in situ stars are found to originate in the earlier disc, at redshifts $\sim 1-1.5$, and subsequently diffusing out of the disc by dynamical heating associated with mergers. The in situ component has a more flattened shape, a net prograde rotation and more metal-rich populations, in quantitative agreement with the observations. We conclude that the dual nature of the stellar halo is entirely compatible with the currently favoured $\Lambda$-CDM model.
\end{abstract}

\section{SPECIFIC PATTERNS OF THE TWO STELLAR HALO COMPONENTS: ACCRETED AND IN SITU}

Over the past few years there have been significant advances in the modelling of stellar haloes of Milky Way-type galaxies in the context of $\Lambda$-CDM cosmology. The majority of these studies have relied on dark matter-only simulations coupled with semi-analytical recipes for assigning stellar mass to infalling satellites (e.g., $[1,5-7])$. These dissipationless models have been most successful at reproducing the properties of the outer $(>20-30 \mathrm{kpc})$ stellar halo and in matching the demographics of the observed tidal streams. However, these models cannot explain the complex chemo-dynamical patterns observed recently in the Solar neighbourhood, from which it has been inferred the existence of two superposed halo components, each with distinctive signatures in spatial distribution, kinematics and chemical abundances. Specifically, the "dual nature" of the stellar halo that has recently emerged from observations entails that one halo component is metal-poor and mostly mixed in phase-space (associated with accretion), and the other one is more metal-rich, flattened and rotating progradely (features that are normally associated with dissipation) (e.g. [2, 3]). Recent hydro-dynamical simulations of Milky Waytype galaxies obtain indeed stellar haloes that have a dual nature $([8,12,16,17])$. Here we use a set of simulated haloes that are in quantitative agreement with the data to infer the potential origin of the two components in the Milky Way halo.

The Galaxies-Intergalactic Medium Interaction Calculation (GIMIC) is a suite of state-of-the-art cosmological hydro-dynamical simulations comprising five spheres of radius $\sim 18 \mathrm{Mpc}$ [4], from which there have been extracted a total of 412 Milky Way-mass galaxies. Font et al. (2011, [8]) and McCarthy et al. (2011, [12]) have shown that these simulations match successfully a broad range of properties of Milky Way and its analogues (e.g. M31). For example, the total stellar masses and luminosities of

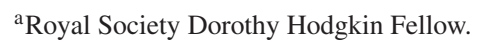

be-mail: af ont@ast.cam.ac.uk

This is an Open Access article distributed under the terms of the Creative Commons Attribution-Noncommercial License 3.0, which permits unrestricted use, distribution, and reproduction in any noncommercial medium, provided the original work is properly cited. 

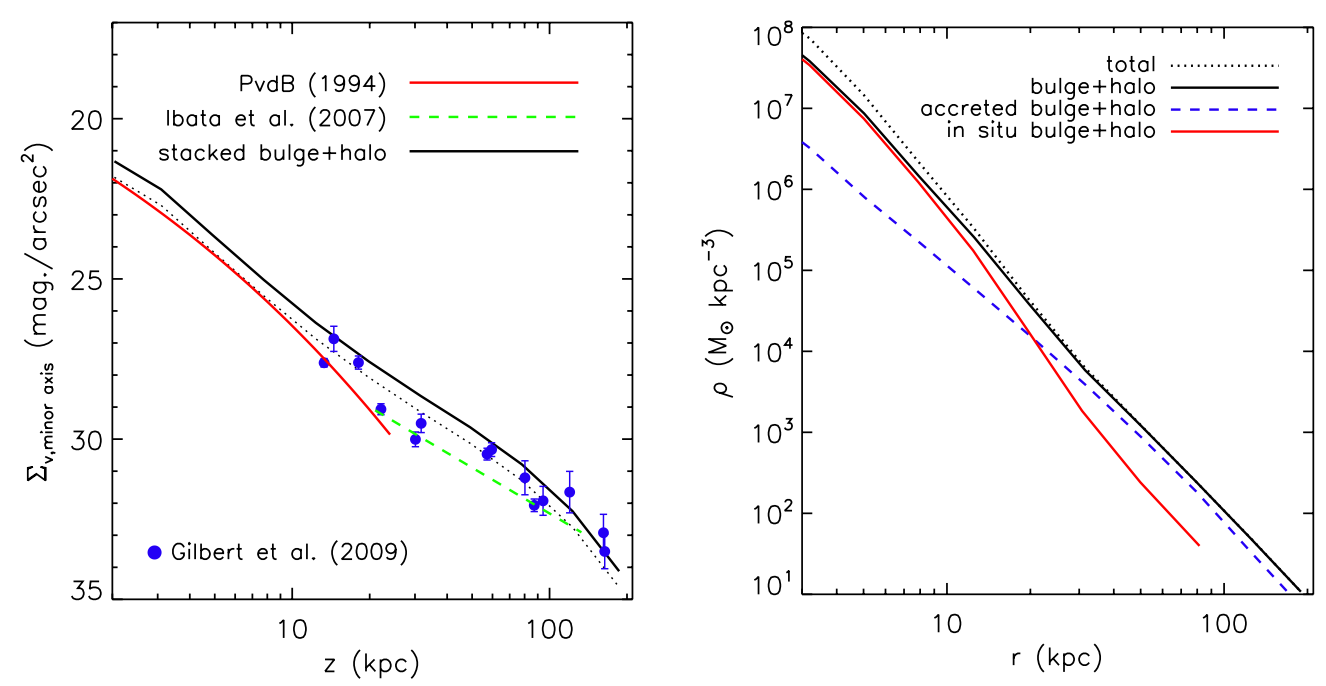

Figure 1. Left: Comparison of the minor axis V-band surface brightness profile for the simulated galaxies with that of M31. The solid black curve represents the stacked profile derived from all 412 simulated disc galaxies. The dotted black curve is the stacked simulated profile shifted down by 0.5 mags $\operatorname{arcsec}^{-2}$. The solid red curve represents the de Vaucouleurs profile fit to the minor axis profile of the M31 spheroid derived by [14]. The dashed green line represents the power-law fit to the outer halo of M31 by [11]. The solid blue circles represent the measurements of [9]. The shape of the stacked profile is in very good agreement with that derived for M31 over a wide range of radii. Right: Median spherically-averaged stellar mass density profiles. The dotted and solid black curves represent all stars and stars in the spheroid only, respectively. The dotted black curve represents all stars (disc + spheroid). The dashed blue curve represents accreted halo stars. The dot-dashed red curve represents spheroid stars that formed in situ.

these galaxies, the rotation velocities and sizes of the stellar discs, and the luminosity functions of satellite galaxies are all in good agreement with the data from Milky Way and M31. Similarly, there is a good match to the spatial distribution of halo stars and their associated chemical abundances [8], while the overall shapes and internal kinematics of the two halo components (accreted and in situ) are matched as well. Some of these properties will be discussed in more detail below. For simplicity, we only show the averaged properties in the sample, which are obtained by stacking all 412 Milky Way-mass galaxies.

Figure 1 compares the averaged V-band surface brightness profiles of the simulated haloes with that of M31. The simulated galaxies are orientated to an edge-on configuration and the profile is calculated along the minor axis, similarly to the M31 data $([9,11,14])$. Quite remarkably, the shape of the stacked simulated profile matches that of the observed surface brightness profile of M31 over almost two decades in radius (from $r \approx 2 \mathrm{kpc}$ to beyond $100 \mathrm{kpc}$ ), including the change in slope from the inner region $\rho \sim r^{-3.5}$ to $\rho \sim r^{-4}$, to the outer region. The right panel shows that the threshold at $\sim 20-30 \mathrm{kpc}$ associated with the change in slope of the mass density/surface brightness corresponds to the transition between the in situ-dominated to the accretion-dominated halo.

Figure 2 shows the stacked Toomre diagrams for a region similat to the of "Solar neighbourhood" ( $R=7.0-10.0 \mathrm{kpc}, Z<2 \mathrm{kpc}$ ). The contours represent averaged velocity dispersions and normalised to $V_{L S R}$. The in situ component shows a clear prograde rotation with a lag ranging from -0.7 to 0.0 (median is $\approx-0.35$ ) times the rotation velocity of the disc. For a disc galaxy with a rotation velocity similar to $\mathrm{M} 31, \approx 240 \mathrm{~km} / \mathrm{s}$, the median lag corresponds to $\approx-84 \mathrm{~km} / \mathrm{s}$. This is similar to the velocity lag reported for the large disc-like structure around M31 by [10]. The accreted component, by contrast, shows a much broader range of velocities, with a non-negligible fraction of stars on retrograde 


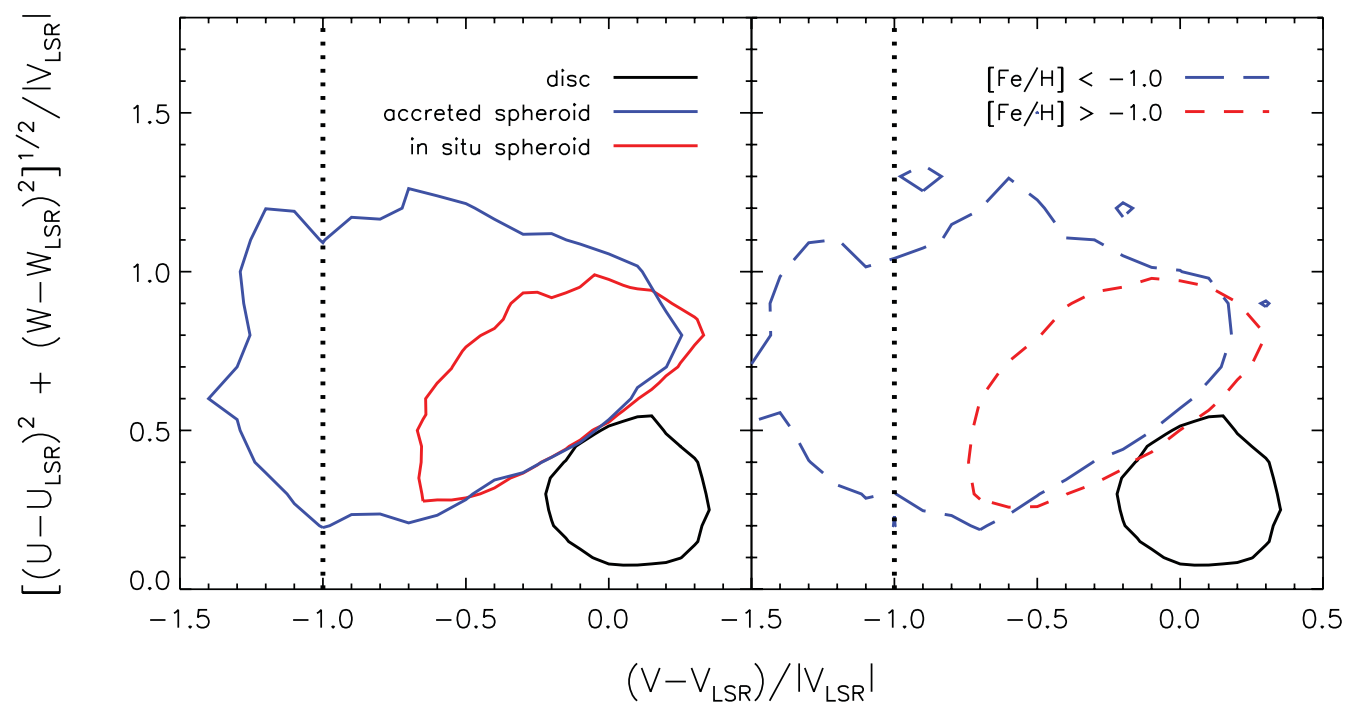

Figure 2. Stacked Toomre diagrams for star particles in the 'Solar neighbourhood' of the simulated disc galaxies. The contours enclose $50 \%$ of the particles for any given subcomponent. Left of the vertical dotted line motion is retrograde with respect to the disc. Relatively metal-rich stars (which are predominantly formed in situ) always rotate in prograde fashion. Relatively metal-poor stars (predominantly accreted) show a much larger range of velocities with a non-negligible fraction on retrograde orbits.

orbits with respect to the disc. The right panel shows a similar dichotomy for the metal-rich stars (predominantly formed in situ) and the metal-poor stars (predominantly accreted). These results are in agreement with the results of [3] based on a sample of stars in the Milky Way Solar neighbourhood. The results are also in qualitative agreement with the kinematics of the two $[\alpha / \mathrm{Fe}]$ halo populations of [13], where high/low $[\alpha / \mathrm{Fe}]$ stars are likely tracers of in situ and accreted components, respectively.

\section{THE ORIGIN OF THE IN SITU' COMPONENT}

Given the remarkable success of GIMIC simulations in matching the properties of the in situ component, one can ask whether they could elucidate further its origin. McCarthy et al. [12] have shown that the origin of the in situ stars can be traced back to the proto-disc of the galaxy. Fig. 3 illustrates the key result that supports this scenario. This shows the fraction of specific kinetic energy in ordered rotation $\left(K_{\text {rot }}\right)$ versus specific total kinetic energy $\left(K_{\text {tot }}\right)$ for in situ stars today compared with the same fraction for the cold gas that generated these stars at $z=z_{\text {obs }}$. The three panels show the tracing back to $z_{\text {obs }}=0.5,1$ and 2, respectively. At any redshift $z_{\mathrm{obs}}$ the cold, star forming gas has a much larger fraction of its energy in highly ordered rotation (e.g., $K_{\text {rot }} / K_{\text {tot }}>0.8$ ) than the corresponding in situ stars at $z=0$. The highest fraction of ordered rotation occurs around $z_{\mathrm{obs}} \sim 1-1.5$ which corresponds to the epoch of disc formation. The majority of present day in situ halo stars formed primarily in a proto-disc at these redshifts. These stars are found to be subsequently ejected from the disc through dynamical heating associated with ongoing mass accretion. This is in general agreement with the scenario proposed by [15] who used, however, idealised (mainly collisionless) simulations of disc bombardment by infalling satellites rather than cosmological hydro-dynamical simulations.

Although in qualitative agreement with the results of Zolotov et al. (2009, [16]; 2010, [17]), the GIMIC simulations differ in several key aspects. The in situ stars in the GIMIC simulations form at radii comparable to where they are presently located, whereas in the Zolotov et al. simulations are formed at significantly smaller radii. Secondly, the in situ population in the GIMIC sample is younger than that 


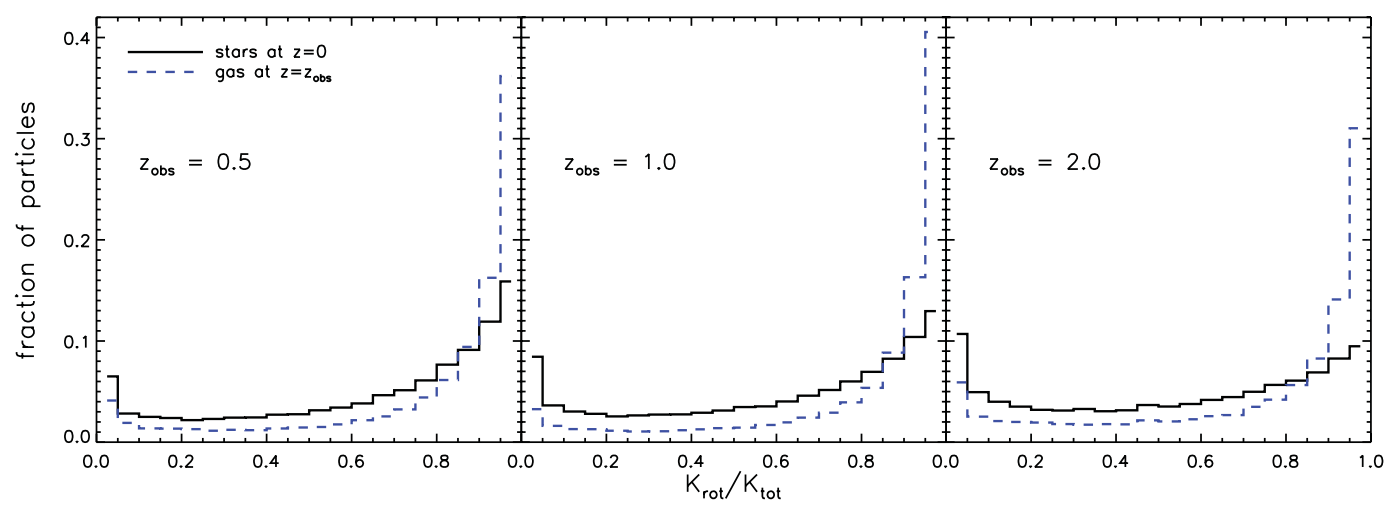

Figure 3. The fraction of kinetic energy in ordered rotation for in situ stars at $z=0$ and the cold gas which formed them at $z=z_{\mathrm{obs}}$. At higher redshift $z_{\mathrm{obs}}$ the cold, star forming gas has a much larger fraction of its energy in highly ordered rotation (e.g., $K_{\text {rot }} / K_{\text {tot }}>0.8$ ) than the stars at $z=0$ which formed from that gas. This difference increases with increasing redshift (up to $z_{o b s} \sim 1.5$ ) and then decreases.

of those galaxies of similar masses in the study of [16]: $z_{\text {form }} \sim 1-1.5$ versus $z_{\text {form }} \approx 3$, respectively. The differences in the formation radii and ages of the in situ stars between the two simulations may be due to the different feedback efficiencies. In the simulations of [16] feedback seems to be relatively less efficient in low-mass haloes, as suggested by the over-production of bright satellite galaxies orbiting Milky Way-mass galaxies today. In order for the in situ stars in the halo to be formed in a proto-disc at relatively late times, an efficient form of feedback is required to operate to delay the formation of these stars. The above differences imply a different formation scenario for in situ stars in the two studies. For the GIMIC sample, [12] show that the in situ stars form primarily from the cooling of hot gas during the initial assembly of the disc, rather than in cold flows, as advocated by [16]. These different formation mechanisms should leave unique imprints in the properties of in situ stars today, such as their spatial and kinematic distribution, and their metallicities. Elucidating the origin of in situ halo stars is crucial for interpreting the data from ongoing and future surveys.

\section{References}

[1] Bullock, J. S., Johnston, K. V., ApJ, 635, (2005) 931

[2] Carollo, D. et al., Nature, 7172, (2007) 1020

[3] Carollo, D., Beers, T. C., Chiba, M. et al., ApJ, 712, (2010) 692

[4] Crain, R. A. et al., MNRAS, 399, (2009) 1773

[5] De Lucia, G., Helmi, A. MNRAS, 2008, 391, 14

[6] Font, A. S., Johnston, K. V., Bullock, J. S., Robertson, B. E., ApJ, 638, (2006) 585

[7] Font, A. S., Johnston, K. V., Bullock, J. S., Robertson, B. E., ApJ, 646, (2006) 886

[8] Font, A. S., McCarthy, I. G., Crain, R. A., Theuns, T., Schaye, J., Wiersma, R. P. C., Dalla Vecchia, C., MNRAS, 416, (2011) 2802

[9] Gilbert, K. M., Font, A. S., Johnston, K. V., Guhathakurta, P., ApJ, 701, (2009) 776

[10] Ibata, R., Chapman, S., Ferguson, A. M. N., Lewis, G., Irwin, M., \& Tanvir, N., ApJ, 634, (2005) 287

[11] Ibata, R., Martin, N. F., Irwin, M., Chapman, S., Ferguson, A. M. N., Lewis, G. F., McConnachie, A. W., ApJ, 671, (2007) 1591

[12] McCarthy, I. G., Font, A. S., Crain. R. A., Schaye, J., Theuns, T., Deason, A. J. MNRAS, (2011), in press; arXiv:1111.1747 
[13] Nissen, P. \& Schuster, A \& A, 511, (2010) L10

[14] Pritchet, C. J., van den Bergh, S., AJ, 107, (1994) 1730

[15] Purcell, C. W., Bullock, J. S., Kazantzidis, S., MNRAS, 404, (2010) 1711

[16] Zolotov, A., Willman, B., Brooks, A. M., Governato, F., Brook, C. B., Hogg, D. W., Quinn, T., Stinson, G., ApJ, 702, (2009) 1058

[17] Zolotov, A., Willman, B., Brooks, A. M., Governato, F., Hogg, D. W., Shen, S., Wadsley, J., ApJ, 721, (2010) 738 